\title{
PHOTOELECTROCHEMICAL CELL WITH DYE MOLECULES ORIENTED IN NEMATIC LIQUID CRYSTAL
}

\author{
N.S. Naser, A. Planner and D. Frackowiak* \\ Institute of Physics, Poznan University of Technology \\ Piotrowo 3, 60-965 Poznan, Poland
}

(Received February 26, 1997; in final form May 5, 1997)

\begin{abstract}
A photoelectrochemical cell consisting of two semiconducting electrodes covered by orienting $\mathrm{SiO}_{x}$ layers and filled with merocyanine solutions in nematic liquid crystal was constructed. Dye molecules were oriented as a result of the guest-host effect. Generation of photocurrent by two components of polarized light gives different values of photocurrent amplitude but anisotropy of photocurrent is lower than that of absorption in the region of a free base form of dye. It is explained by dominant participation of a protonated form of dye in the photocurrent generation. The last form exhibits a lower degree of orientation than the free base form of dye.
\end{abstract}

PACS numbers: 42.70.Df, 42.79.Kr, 61.30.Gd

\section{Introduction}

The purpose of the investigation was to construct a photoelectrical sensor simple and sensitive to the polarization of light. Such sensor together with a similar one but measuring the whole light intensity can be used for the monitoring of the scattering of the probe light beam. This type of measurements can be of value for investigations of the chemical or photochemical reactions in which small crystals or colloidal particles are formed.

The photoelectrochemical cell used in our investigations was composed of the solution of stilbazolium merocyanine dye in nematic liquid crystal located between two transparent semiconducting electrodes covered by orienting layer. As a result of the interaction of liquid crystal with the orienting layer the liquid crystal molecules are oriented [1-3]. Due to the guest-host effect also the dye molecules are uniaxially oriented along the axis located in the plane of the electrodes [1-3], in such a system, as it was shown previously, the degree of orientation of stilbazolium merocyanines is high [4].

\section{Materials and methods}

Figure 1 shows the chemical formulae of stilbazolium merocyanines used (1-(11'-hydroxyundecyl)-4-/(3,5-di-tert-butyl-4-oxocyclohexa-2,5-dienylidene)

*Corresponding author. 
ethylidene/-1,4-dihydropyridine) and (1-hexadecyl-4-/(3,5-di-tert-butyl-4-oxocyclohexa-2,5-dienylidene)ethylidene/-1,4-dilhydropyridine) named further in the text as mero-E and mero-F, respectively. The formula of nematic liquid crystals (LC) k15 (4-cyano-4'-pentylobiphenyl), the $N$-(4-methoxybenzylidene)-4'-butylaniline (MBBA) and $N$-(4-ethoxybenzylidene)-4'-butylaniline (EBBA) are also shown in Fig. 1. The liquid crystal k15 (named also as 5CB) was from E. Merck (Darmstadt, Germany), MBBA and EBBA were from Aldrich Chemical Company (Steinheim, Germany). Liquid crystals were used without further purification. Two types of LC solvents: $\mathrm{k} 15$ and the mixture of MBBA + EBBA (3:2 w/w) were used. Merocyanines were obtained from Prof. Ilona Gruda (Trois Rivieres, Canada). The synthesis and purification of dyes were described in Refs. [5-7].

a)

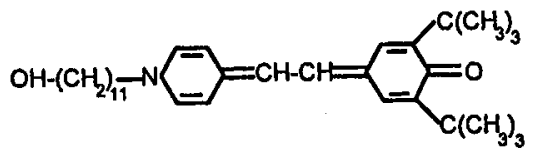

b)

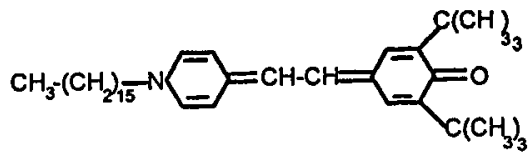

c)

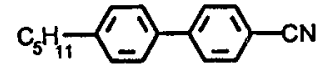

d)

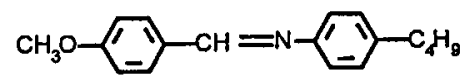

e)

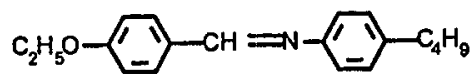

Fig. 1. Structural formula of the investigated dyes and liquid crystals: (a) mero-E, (b) mero-F, (c) k15, (d) MBBA, (e) EBBA.

The semiconducting layer of $\mathrm{In}_{2} \mathrm{O}_{3}$ was first deposited on Glastron (France) glass plate, followed by the deposition of the $\mathrm{SiO}_{x}$ orienting layer. From two such windows and teflon spacer (thickness $60 \mu \mathrm{m}$ ) a cell filled with $10^{-2} \mathrm{M}$ or $10^{-3} \mathrm{M}$ solution of merocyanine in LC was constructed. The polarized absorption of the cells was measured with a M40 Specord (Carl Zeiss, Jena, Germany) spectrophotometer equipped with polarizers and sample holders. As a reference an unpigmented cell with LC was used. The photogenerated current kinetics and current-voltage dependences (voltammograms) were measured.

\section{Results and discussion}

Figure 2 shows polarized absorption spectra of the investigated cells with dyes in $\mathrm{k} 15$ and an example of absorption of mero-E in MBBA + EBBA. Polarized spectra of merocyanines in MBBA + EBBA were reported previously [4]. The 
maximum at about $400 \mathrm{~nm}$ belongs to a protonated form of dye whereas both maxima in a 500-700 $\mathrm{nm}$ spectra range belong to a free base form of dye [5-7]. The absorption of the protonated form in a region of $380-420 \mathrm{~nm}$ for both dyes in $\mathrm{k} 15$ is low but for mero-F (Fig. $2 \mathrm{~b}$ ) a concentration ratio of the protonated to the free base absorption is higher than for mero-E. In MBBA + EBBA the dyes are in a much higher degree protonated as it follows from Fig. 2c and from the previous results $[4,8,9]$.

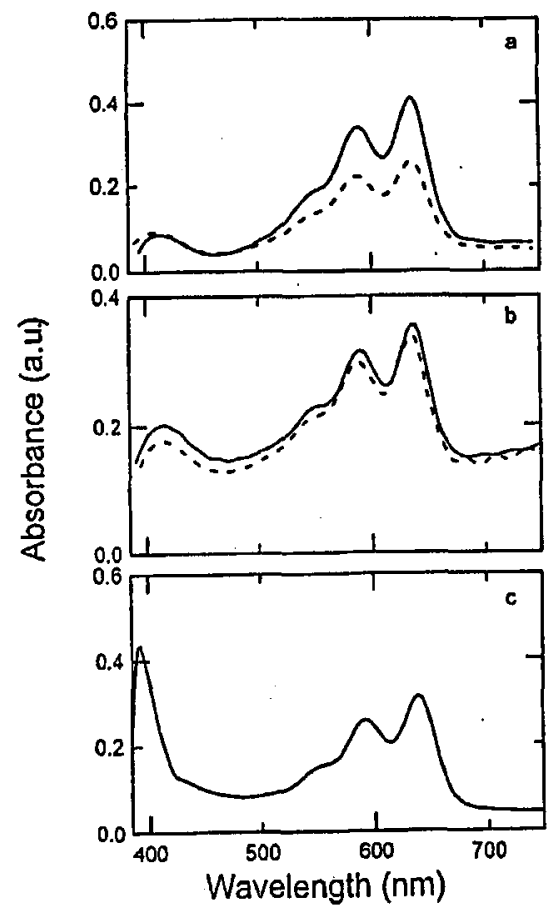

Fig. 2. Absorption spectra of: (a) mero-E in $k 15$ [a continuous line shows the parallel component and a broken line shows the perpendicular component], (b) mero-F in k15 [components as in (a)], (c) mero-E in MBBA + EBBA (natural light).

The orienting factor (degree of orientation) $s=A_{\|}-A_{\perp} / A_{\|}+2 A_{\perp}$ was calculated with respect to the axis of the highest orientation of dye. In order to establish the direction of this axis, light polarized vertically (along the longer cell dimension) was transmitted by the cell which was then rotated at various $(\theta)$ angles with respect to the vertical position. As it follows from Fig. 3 the preferential orientation of the dye long axis (and parallel to its transition moments of absorption) is located at $(\theta)$ about 60 degrees with respect to the longer cell side. Because the merocyanine dyes are usually oriented parallel to the LC orientation axis [4] it is probably also the direction of the LC orientation.

The orientation factors for two dyes for various absorption maxima are gathered in Table I. These values depend on a type of LC, a type of dye and a spec- 


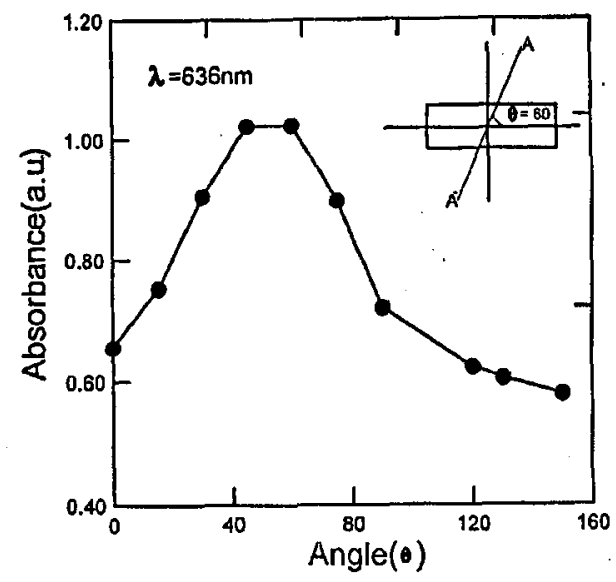

Fig. 3. The dependence of absorbance of a cell on the angle $\theta$ (explained in the text and in the inset scheme showing the cell and the dye orientation axis $A A^{\prime}$ ).

\section{TABLE I}

Absorption anisotropy of mcrocyanines in LC (k15) (explanation in the text).

\begin{tabular}{c|c|c|c|c}
\hline \hline Dye & Maxima $[\mathrm{nm}]$ & $s$ & $\beta^{0}$ & $A_{\|} / A_{\perp}$ \\
\hline Mero-E & 412 & -0.013 & 55.27 & 0.96 \\
& 589 & 0.151 & 48.79 & 1.53 \\
& 636 & 0.165 & 48.20 & 1.59 \\
\hline Mero-F & 418 & 0.043 & 53.70 & 1.13 \\
& 589 & 0.014 & 54.20 & 1.04 \\
& 637 & 0.010 & 54.30 & 1.03
\end{tabular}

tral region investigated. For example for mero-E in $\mathrm{k} 15$ the maximum at $636 \mathrm{~nm}$ $s=0.165$ and for the same dye for the maximum at $589 \mathrm{~nm} s=0.151$ whereas for the protonated form it equals $s=-0.0133$. The orientation factors of both maxima belonging to the free base form of dye are similar which supports our previous conclusion [8] that these maxima represent two vibronic transitions of the same form. The angles $(\beta)$ between the direction of the transition moments of absorption and the direction of the preferential orientation axis calculated from the formula $s=\left(3 \cos ^{2} \beta-1\right) / 2$ are also similar for both free base forms whereas for the protonated form is strongly different (Table I). The degree of orientation of mero-F is much lower than that of mero-E. It means that longer molecules are in a lower degree oriented in the LC used. It is a similar result as it was found previously for other merocyanines [4].

Figure 4 shows the kinetics of the photocurrent generation after switching light on and the decay after switching light off for two polarizations of light in the case of mero-E in $\mathrm{k} 15$. The ratio of the amplitudes of photocurrent at par-. 


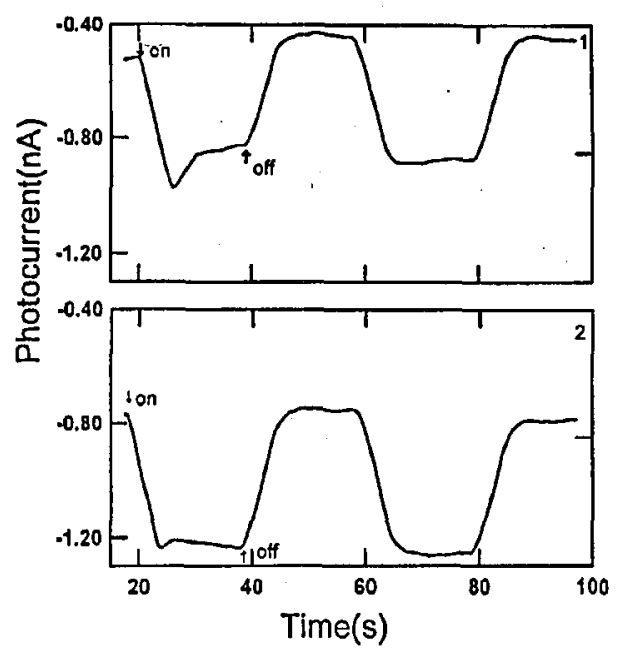

Fig. 4. The kinetics of photocurrent generated by polarized light for mero- $\mathrm{E}$ in $\mathrm{k} 15$ (1-perpendicular component, 2 - parallel component).

allel light polarization to the one at perpendicular is 1.12 , whereas the ratios of absorption of two polarized components are in the free base region about 1.5. The ratio of polarized absorption components in a band of the protonated form is 0.96 (Table I). Kinetics for mero-F in $\mathrm{k} 15$ are similar to these for mero-E. In the case of mero- $\mathrm{F}$ the ratio of photocurrent components, equal to 1.10 , is more similar to the ratio of absorption components than in the case of mero-E (Table I). Because of the similarity of the anisotropy ratio of the protonated form absorption band and photocurrent one can suspect that the predominantly protonated band is responsible for the photocurrent generation. Therefore, the photocurrent generated by the light transmitted by two filters was measured. The one filter was cutting light absorbed by the protonated form, the second one was transmitting this spectral region of light. The ratio of the surface illumination intensities in the spectral range $340-470 \mathrm{~nm}$ used and in the region of $470-700 \mathrm{~nm}$ is about 0.65 . When only the free base form was illuminated the photocurrent was not measurable. It shows that rather the protonated form is responsible for the photocurrent. In order to confirm this supposition the amplitudes of photocurrents for the same dye but in two solvents in which the dye is in a different degree protonated (in $\mathrm{k} 15$ and MBBA + EBBA) were compared. The experiment was repeated for several pairs of cells with these two solvents but the differences between cells with the same solvent were comparable with the differences between the pair of cells. It seems that the influence of such parameters as the quality of conducting and orienting layers, or humidity of the LC on the amplitude of photocurrent is comparable to the effect due to the concentration of the protonated form.

A dependence of maximal photocurrent on the acting light intensity is presented in Fig. 5. As it follows from this figure, this is not a linear dependence. Because the light intensity is low enough to avoid nonlinear effects the shape of 


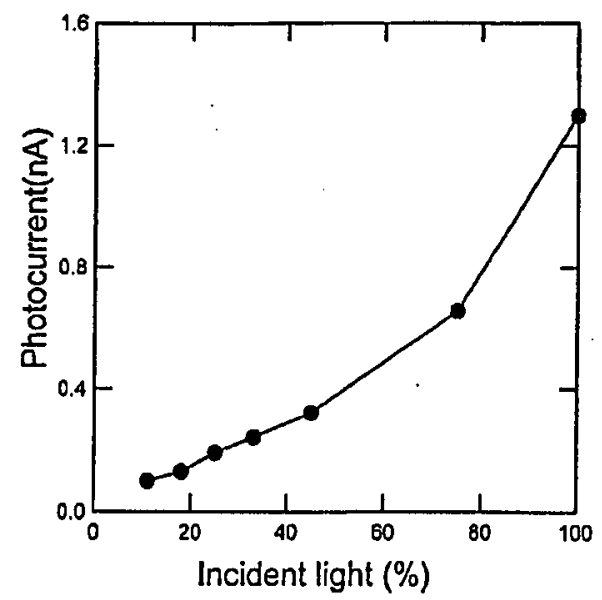

Fig. 5. The dependence of measured photocurrent on incident light intensity (\%) measured for mero-E in k15.

this curve suggests that, as was previously found [9], several processes such as perturbation of space charge layers and reorganization of LC matrix, characterized by different kinetics are contributing to the current generation. The ratios of the contributions from various processes could be different at various light intensities.

Current-voltage dependences (voltammograms) taken at different speeds of voltage changes (given in captions) are shown in Fig. 6 for mero-E and mero-F. Both measurements have been done in $\mathrm{k} 15$. Previously [4] similar measurements

TABLE II

The electrical parameters of photoelectrochemical cells with merocyanines in k15 (obtained from $I(V)$ dependences).

\begin{tabular}{c|c|c|c|c|c}
\hline \hline Dye & \multirow{2}{*}{$\begin{array}{c}\text { Speed of voltage } \\
{[\mathrm{mV} / \mathrm{s}]}\end{array}$} & \multicolumn{2}{|c|}{ Capacity $\left[10^{-6} \mathrm{~F}\right]$} & \multicolumn{2}{c}{ Resistance $\left[10^{6}\right.$ ohm $]$} \\
\cline { 3 - 6 } & & Light on & Light off & Light on & Light off \\
\hline Mero-E & 10 & 0.14 & 0.13 & 6.89 & 7.69 \\
& 50 & 0.13 & 0.11 & 6.02 & 6.80 \\
& 100 & 0.11 & 0.10 & 5.20 & 6.36 \\
\hline Mero-F & 50 & 0.24 & 0.14 & 0.90 & 0.93 \\
& 100 & 0.19 & 0.15 & 0.90 & 0.93 \\
& 200 & 0.15 & 0.14 & 0.90 & 0.91
\end{tabular}

were done for merocyanines in LC mixture MBBA + EBBA in which the dye was in a very high degree protonated. In both solvents (MBBA + EBBA and k15) $I(V)$ characteristics are not olımic by nature, i.e. the cells have the semiconductor-LC junction. But in the case of $\mathrm{k} 15$ a "hysteresis" loop is broader, at the same speed of the voltage changes as in the case of MBBA + EBBA. The unpigmented LC gives 


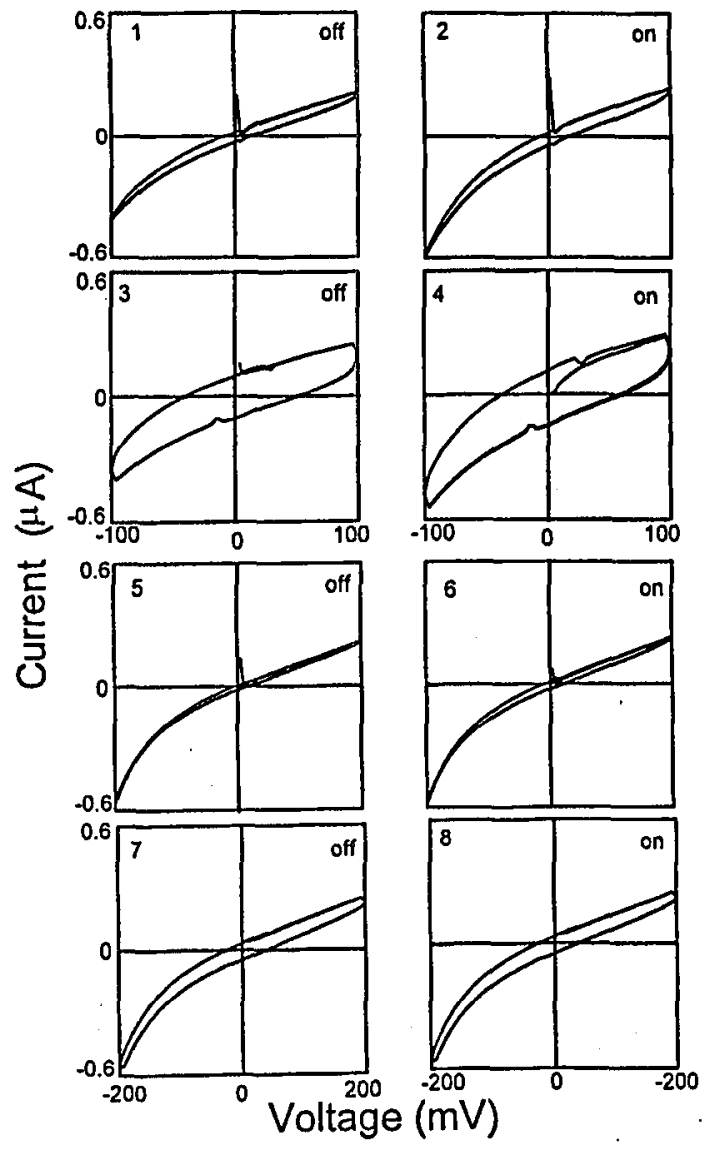

Fig. 6. Voltage-current dependence for various speeds $(\mathrm{d} u / \mathrm{d} t)$ of voltage increase for dark (off) and illuminated (on) cells:

1-4 mero-E in $\mathrm{k} 15$ :

$1-\mathrm{d} u / \mathrm{d} t=10 \mathrm{mV} / \mathrm{s}$ (light off), $2-\mathrm{d} u / \mathrm{d} t=10 \mathrm{mV} / \mathrm{s}$ (light on), $3-\mathrm{d} u / \mathrm{d} t=$ $100 \mathrm{mV} / \mathrm{s}$ (light off), $4-\mathrm{d} u / \mathrm{d} t=100 \mathrm{mV} / \mathrm{s}$ (light on);

$5-8$ mero- $F$ in $\mathrm{k} 15$ :

$5-\mathrm{d} u / \mathrm{d} t=50 \mathrm{mV} / \mathrm{s}$ (light off), $6-\mathrm{d} u / \mathrm{d} t=50 \mathrm{mV} / \mathrm{s}$ (light on), $7-\mathrm{d} u / \mathrm{d} t=$ $200 \mathrm{mV} / \mathrm{s}$ (light off), $8-\mathrm{d} u / \mathrm{d} t=200 \mathrm{mV} / \mathrm{s}$ (light on).

also non-ohmic $I(V)$ but with a very narrow loop [4]. The illumination of the cell causes the change in $I(V)$ curves (Fig. 6). On the basis of $I(V)$ dependences the capacities and resistances of the cell according to the method given in Ref. [8] were calculated and gathered in Table II. $\Lambda$ s it follows from this table the resistances of the cells are decreasing and capacities are increasing as a result of the cell illumination. The changes of capacity due to the cell illumination are larger at a slow speed than at a higher one, especially in the case of mero- $F$. The differences can be related with different lengths of the dye molecule. Mero- $F$ is longer than 
mero-E. Reorientation due to the charge generated by the illumination could be different for these two molecules. The loops are broader at a higher speed of a voltage increase. At a slow speed changes are equilibrated and the loop became narrow. It suggests that rather quick processes occurring in hundredths of seconds are responsible for the observed $I(V)$ hysteresis. The kinetics depends on a type of dye. Loops are broader for shorter dye molecules.

\section{Conclusions}

The investigated dyes exhibit a certain dependence of the photocurrent intensity on the light polarization, but because the predominantly protonated form, which is in a lower degree oriented than the free base form, is responsible for the photopotential generation, therefore this dependence is not very strong. The anisotropy effects depend on a type of dye as well as on a type of solvent which strongly influences the dye protonation. All investigated cells exhibit the non-ohmic $I(V)$ dependence, high resistance (of order $10^{6} \mathrm{ohm}$ ) and the capacity which is increasing as a result of the illumination. This shows that the charge space distribution is changed as a result of illumination. The low anisotropy of electric signal can be also due to the lower orientation of dye in the vicinity of the electrode than in the whole volume of the cell. Absorption gives averaged orientation, whereas the photocurrent generation is infuenced only by the orientation of the thin layer of pigmented LC located at the electrode [9].

The present result shows that it is possible to construct a photoelectrical sensor sensitive on the light polarization, but the parameters of dye and solvent have to be carefully chosen.

\section{Acknowledgment}

This work was supported by the Poznan University of Technology grant No. $62-133 / 2$ (1997).

\section{References}

[1] G.H. Brown, J.J. Wolken, Liquid Crystal and Biological Structure, Academic Press, New York 1979.

[2] A. Adamczyk, The Surface Interaction in Electrical and Optical Processes in Liquid Crystalline Thin Layer, Warsaw Technical University, Warsaw 1979 (in Polish).

[3] R. Subramanian, L.K. Patterson, II. Levanon, Photochem. Photobiol. 41, 511 (1985).

[4] J. Goc, D. Frackowiak, J. Photochem. Photobiol. A, Chem. 59, 233 (1991).

[5] I. Gruda, S. Hotchandani, D. Frąckowiak, Photobiochem. Photobiophys. 12, 267 (1986).

[6] I. Gruda, F. Bolduc, J. Org. Chem. 49, 3300 (1984).

[7] M. Niedbalska, I. Gruda, Can. J. Chem. 68, 691 (1990).

[8] D. Frąckowiak, I. Gruda, M. Niedbalska, M. Romanowski, A. Dudkowiak, J. Photochem. Photobiol. A, Chem. 54, 37 (1990).

[9] A. Ptak, A. Der, R. Toth-Boconadi, N.S. Naser, D. Frąckowiak, J. Photochem. Photobiol. A, Chem., accepted. 